\title{
Pharmacists' care of diabetes patients during Ramadan
}

\author{
Kerry Wilbur ${ }^{1}$
}

Received: 24 December 2014/ Accepted: 4 March 2015/Published online: 20 March 2015

(C) Koninklijke Nederlandse Maatschappij ter bevordering der Pharmacie 2015

It was encouraging to read the recent report of "Community pharmacists' knowledge of diabetes management during Ramadan in Egypt" by Amin and Chewning [1] highlighting the potential for pharmacists to fulfill this public health education role. In the Middle East in particular, this is a prevalent aspect of diabetes care, not only during Ramadan, but also during other periods patients choose to fast throughout the year.

In fact, ours was the first published experience of pharmacist's roles in this regard [2]. Like the authors, we assessed pharmacist understanding of appropriate care with a series of case scenarios as part of a broader questionnaire seeking to determine Qatar pharmacists' knowledge, practice and attitudes towards guiding diabetes patient management during Ramadan. As indicated by the researchers who read our conference abstract, we also found capacity to correctly advise on appropriate insulin dose adjustments in fasting patients to be lowest when compared to responses regarding oral therapy. However, our sample was smaller $(\mathrm{n}=178)$ and included pharmacists working in hospital settings (41\%). The discrepancy found in Amin and Chewning's paper relating to pharmacists' confidence in (high) and actual scored knowledge (low) related to medication dosing adjustments could be influenced by the survey administration methodology; subjects may actually knowingly overestimate their abilities when faced with the interviewer who is taking the responses (social desirability bias) [3].

We agree with these authors that more continuing professional development is desired by and should be offered to practicing pharmacists in the management of diabetes patients during Ramadan, coupled with augmented emphasis in undergraduate curriculums in the region. Future knowledge assessments would also benefit from inclusion of dosing consideration for the expanding treatment alternatives in diabetes (thiazolidinediones, dipeptyl-4-inhibitors, sodium-glucose transporter 2 inhibitors) and the management of medication for the concomitant diseases these patients most often have [4].

\section{References}

1. Amin MEK, Chewning B. Community pharmacists' knowledge of diabetes management during Ramadan in Egypt. Int J Clin Pharm. 2014;36:1213-21.

2. Wilbur K, Al Tawengi K, Remoden E. Diabets patient management by pharmacists during Ramadan. BMJ Health Serv Res. 2014; 14:117-23.

3. Furnham A. Response bias, social desirability and dissimulation. Personal Individ Differ. 1986; 7:385-400.

4. Al Hreashy FA, Mobierek AF. Prescription practice for diabetes management among a female population in primary health care. Int J Family Med 2014; 2014.

\footnotetext{
Kerry Wilbur

kerrywilbur.is@gmail.com

1 College of Pharmacy, Qatar University, Doha, Qatar
} 